\title{
On the Interpretation of Nonresonant Phenomena at Colliders
}

\author{
Miguel G. Folgado $\mathbb{D}^{1}$ and Veronica Sanz $\mathbb{D}^{1,2}$ \\ ${ }^{1}$ Instituto de Física Corpuscular (IFIC), Universidad de Valencia-CSIC, E-46980 Valencia, Spain \\ ${ }^{2}$ Department of Physics and Astronomy, University of Sussex, Brighton BN1 9QH, UK
}

Correspondence should be addressed to Miguel G. Folgado; migarfol@ific.uv.es

Received 24 August 2020; Accepted 23 February 2021; Published 17 March 2021

Academic Editor: Diego Saez Chillon Gomez

Copyright (c) 2021 Miguel G. Folgado and Veronica Sanz. This is an open access article distributed under the Creative Commons Attribution License, which permits unrestricted use, distribution, and reproduction in any medium, provided the original work is properly cited. The publication of this article was funded by SCOAP ${ }^{3}$.

\begin{abstract}
With null results in resonance searches at the LHC, the physics potential focus is now shifting towards the interpretation of nonresonant phenomena. An example of such shift is the increased popularity of the EFT programme. We can embark on such programme owing to the good integrated luminosity and an excellent understanding of the detectors, which will allow these searches to become more intense as the LHC continues. In this paper, we provide a framework to perform this interpretation in terms of a diverse set of scenarios, including (1) generic heavy new physics described at low energies in terms of a derivative expansion, such as in the EFT approach; (2) very light particles with derivative couplings, such as axions or other light pseudoGoldstone bosons; and (3) the effect of a quasicontinuum of resonances, which can come from a number of strongly coupled theories, extradimensional models, clockwork set-ups, and their deconstructed cousins. These scenarios are not equivalent despite all nonresonance, although the matching among some of them is possible, and we provide it in this paper.
\end{abstract}

\section{Why Nonresonant Phenomena}

At the Large Hadron Collider (LHC), a tremendous effort has been devoted to searches for new states, which in their simplest form would manifest as resonances, an excess of events in a narrow kinematic range. Alas, direct searches have provided no fruit so far, diminishing hopes that the LHC would discover new particles connected to the electroweak scale.

At the same time, LHC experiments have continued improving their understanding of the data and enabled an unprecedented precise characterization of Standard Model (SM) particles. Particularly impressive is the evolution of the Higgs characterization, moving from discovery to precision measurements in a few years. In Figure 1, we can appreciate the level of effort made to contrast precise measurements of the LHC experiments and our best theoretical understanding.

The lack of discoveries is certainly a frustrating aspect of the LHC results, but with improved precision, a new opportunity opens up. The same SM particles whose characterization is so carefully accomplished could provide hints for new phenomena beyond the SM. Among all the SM particles, high hopes are placed on heavy particles, more closely con- nected to the electroweak symmetry breaking sector: the Higgs particle, massive vector bosons, and third-generation fermions. Were their properties deviate from the SM predictions, we might be able to trace back these deviations to new physics. This indirect route may be the key to enter new sectors in Nature.

These two approaches of searching for new physics, direct search for new states and indirect search for new effects in known SM particles, complement each other. There is a wide range of new theories of Nature which at the LHC energies do not show up as narrow resonances, and for those, the best way to find them is by looking at excesses in tails of kinematic distributions.

The aim of this paper is to provide a framework to interpret nonresonant phenomena in terms of a diverse set of scenarios. We will provide a number of well-motivated models which, in some area of their parameter space, lead to no resonances but excesses in tails.

The first paradigm we will discuss is the Effective Field Theory (EFT) approach, suitable for theories with resonances too heavy to be accessed directly at the LHC and only showing up as virtual effects. 

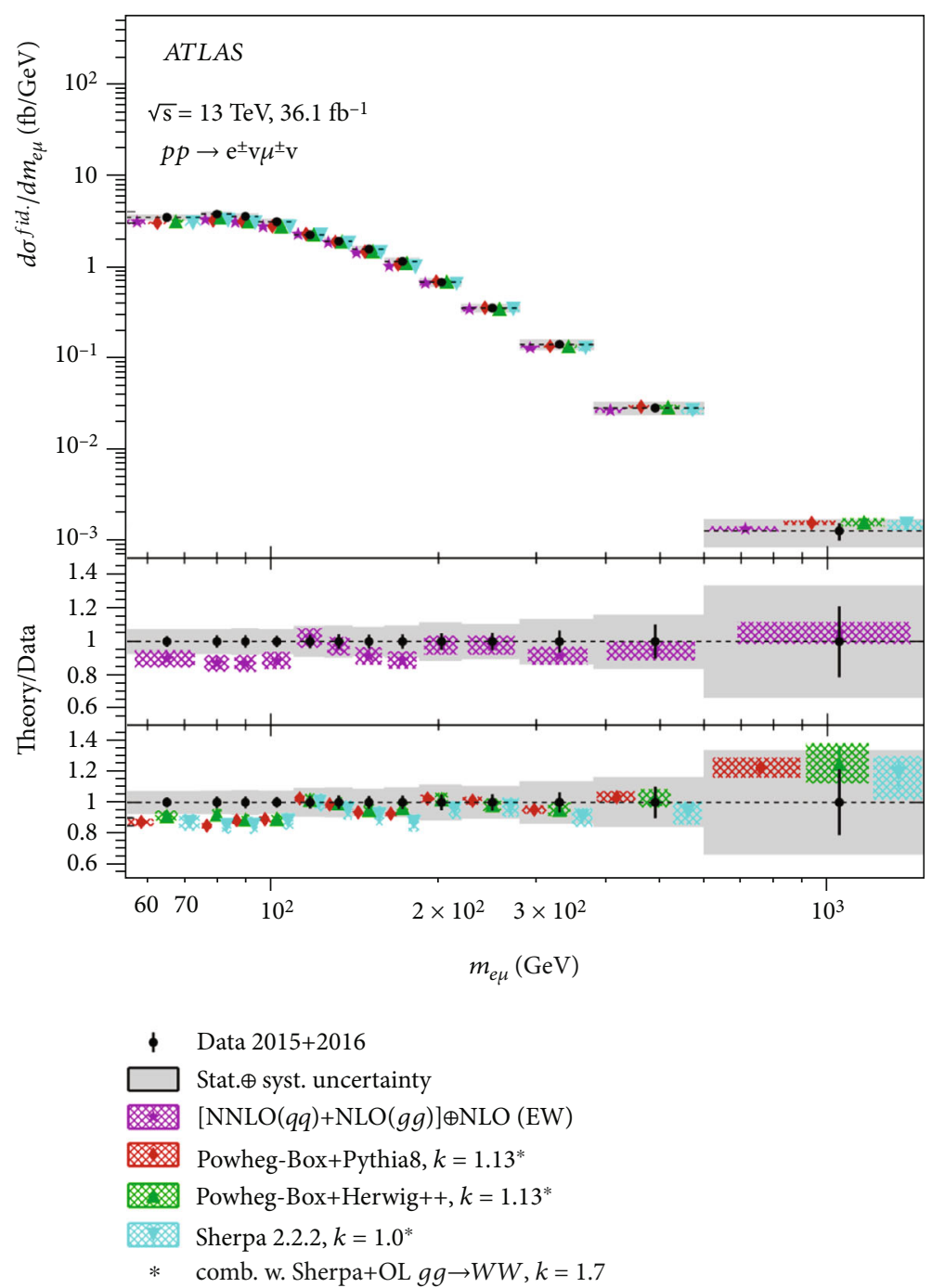

Figure 1: An example of a precise determination of a kinematic distribution (invariant mass of the two leptons in $W^{+} W^{-}$production) and its comparison with different theoretical calculations and showering tools. Figure from Ref. [1].

The EFT is a well-known and nowadays a rather standard way of organizing nonresonant searches, but it is certainly not the only one. There are other interesting theories which exhibit the same experimental signatures. One class of such models is represented by many close-by resonances forming a quasicontinuum in $\sqrt{\hat{s}}$, the parton energy. This type of behaviour does appear in many extensions of SM which predict towers of resonances with the same quantum numbers but increasing mass. Were these towers broad/close-by, they would lead to nonresonant tails. Typical examples of this type of phenomena appear in theories with new strong interactions, extradimensions, or clockwork theories, to name a few.

Another distinct set of theories which could only be discovered via nonresonant searches are scenarios with light particles, so light that usual trigger cuts would prevent us from reaching their resonant kinematic regions but could still be discovered by their off-shell production. If the coupling of these light states to SM particles was derivative, the off-

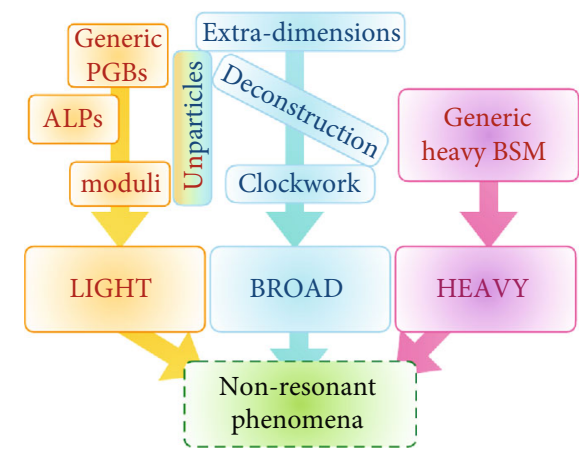

Figure 2: Schematic view of models of nonresonant behaviour.

shellness could be compensated by the natural increase of the cross-section. A paradigmatic example of this kind of scenarios is an axion or axion-like particle coupled to SM gauge bosons (see Ref. [2] for a discussion of this behaviour). 


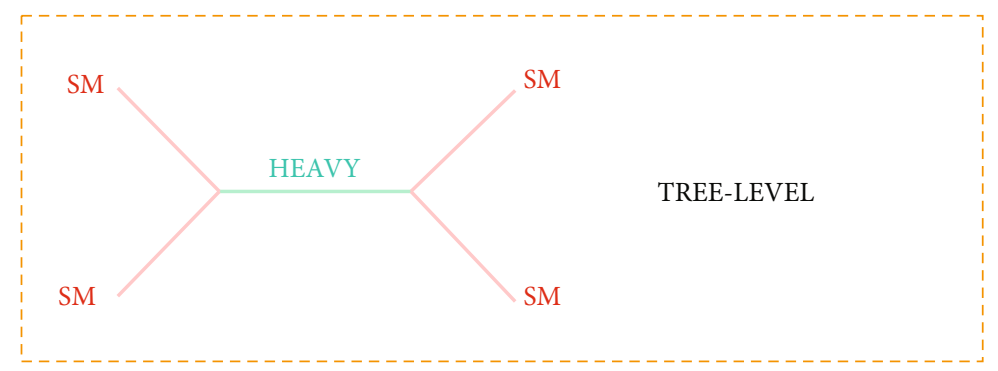

(a)

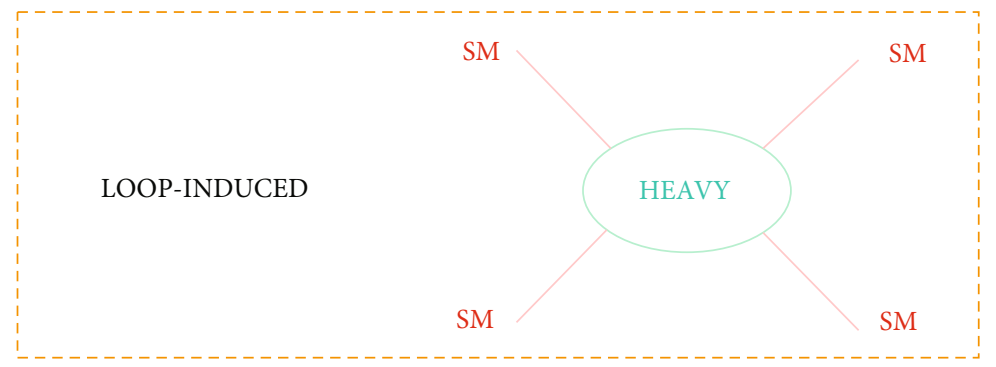

(b)

FIGURE 3: Sketch of the UV origin of EFT effects.

The paper is organized as follows. In Section 2, we discuss the models which can provide a basis for experimental interpretation. In Section 3, we discuss parton-level properties of the cross-sections and provide a dictionary to match parameters among the models which share common experimental tails. We also discuss how these parton-level results generalize to a total production cross-section. Finally, in Section 4, we summarise our findings.

\section{Models of Nonresonant Behaviour}

In this section, we describe new physics scenarios which lead to nonresonant behaviour, as well as relations among them (see Figure 2). Broadly speaking, these scenarios fall into three categories:

(1) Heavy but narrow new states, due to some new sector well above the electroweak scale

(2) Broad/nearby states at the electroweak scale

(3) Light states, with mass well below electroweak's, and with derivative couplings

Many models of new physics at some scale or in some range of their parameter space could show up at colliders as nonresonant excesses. In the rest of the section, we provide some context for some of the most popular models in each category. In the next section, we will provide a dictionary to relate scenarios of broad/nearby resonances, hence unifying their interpretation.

2.1. Heavy Physics: The Effective Field Theory (EFT) Case. We start with the paradigmatic example of EFTs. In this framework, one studies theories which predict a new sector of resonances coupled to the SM, with masses higher than the energies one is able to probe with current experiments. These new resonances will not be produced as final on-shell states but can influence the behaviour of SM particles through their virtual effects.

One could consider the effect of new resonances on a scattering or decay process via tree-level (Figure 3(a)) or loop (e.g., Figure 3(b)) exchange of the heavy states. As the resonances are heavy, $M \gg \sqrt{\widehat{s}}$, one can understand the impact of the new sector as an expansion in energy over mass with various Lorentz structures involving just SM states as dynamical.

For example, let us consider classes of models which affect the electroweak symmetry breaking sector of the SM. Their presence would modify the interactions of the Higgs and massive gauge bosons, e.g., via new types of interactions described by new operators in the Lagrangian such as

$$
\bar{c}_{H W} \frac{2 g}{m_{W}^{2}}\left(D^{\mu} H^{\dagger}\left(T_{2}\right)_{k} D^{\nu} H\right) W_{\mu \nu}^{k}
$$

where $H$ is the Higgs doublet and $W_{\mu \nu}$ is the $S U(2)_{L}$ field strength. These new types of interactions are formally nonrenormalizable and grow with the energy of the scattering process. At the level of Higgs-vector boson interactions, they will modify the coupling structure as [3]

$$
\begin{aligned}
& {\left[h\left(p_{1}\right), W^{\mu}\left(p_{2}\right), W^{\nu}\left(p_{3}\right)\right]} \\
& \quad=\operatorname{igm}_{W}\left[\eta^{\mu \nu} \frac{\bar{c}_{H W}}{m_{W}^{2}}\left(2 p_{2} \cdot p_{3}+\left(p_{2}^{2}+p_{3}^{2}\right)-2 p_{2}^{v} p_{3}^{\mu}-\left(p_{2}^{\mu} p_{2}^{v}+p_{3}^{\mu} p_{3}^{v}\right)\right]\right.
\end{aligned}
$$

These new types of interactions grow with energy and exhibit different angular dependence than the simple SM $\eta^{\mu \nu}$ structure. They will lead to excesses in high momentum tails of production of SM gauge and Higgs bosons, such as $W^{+} W^{-}$distributions shown in Figure 1 . The behaviour of 
this tail, the rate at which it grows with energy, will be dictated by the form of the operator in Equation (1) and the value of its coefficient $\bar{c}_{H W}$, but their interpretation in terms of new sectors of Nature could be very different.

For example, in Ref. [4], we computed three possible interpretations of these effects in terms of new scalars coupled to the electroweak sector:

$$
\begin{aligned}
\bar{c}_{H W} & \simeq \lambda_{h S}^{2} \frac{v_{s}^{2} v^{2}}{m_{S}^{4}}\left(S U(2)_{L} \text { Singlet }\right) \simeq-\frac{2 \lambda_{h H}}{192 \pi^{2}} \frac{m_{W}^{2}}{m_{H_{2}}^{2}}\left(S U(2)_{L} \text { Doublet }\right) \\
& \simeq-\frac{c_{\mathrm{CFT}}}{4} \frac{m_{h}^{2} v^{2}}{f^{2} m_{r}^{2}}(\text { Radion/Dilaton })
\end{aligned}
$$

where $m_{S, H_{2}, r}$ are the singlet, doublet, and radion/dilaton masses, $\lambda_{h S, h H}$ are the singlet/doublet-Higgs couplings, $v_{S}$ is the singlet vev, and $c_{\mathrm{CFT}}$ is a parameter of the new quasiconformal sector (see Ref. [4] for details, as well as Ref. [5] to understand how the singlet expression has been obtained).

Despite sharing a common experimental search (same cuts and statistical procedure), the limit on the value of $\bar{c}_{H W}$ would translate into very different limits for the three scenarios discussed above. Moreover, a combined measurement in more than one diboson channel, e.g., $W W, Z Z, Z \gamma$, and $\gamma \gamma$, could be used to disentangling the three different models (see, e.g., the discussion in Ref. [6] along these lines).

Moreover, for some of these scenarios, the EFT procedure may not be a valid approach. This theoretical limitation is sometimes denoted by the generic question of the range of validity of the EFT (see, e.g., Refs. [7-10]). For example, if one were to use the distribution in Figure 1 to set limits on a value of $\bar{c}_{H W}$, the limit would be mostly dominated by the highest $m_{e \mu}$ bins, but if this limit led to a model interpretation with particle masses $M<m_{e \mu}$, the EFT approach would break down.

\subsection{Light New Physics: Axions and Other Pseudo-Goldstone} Bosons. Since Goldstone obtained his theorem, we know that light degrees of freedom (Goldstone bosons) are a manifestation of a global symmetry which is spontaneously broken by some UV dynamics, e.g., confinement from a strong gauge sector. We know this description to be very predictive in the low-energy sector of QCD $(\chi \mathrm{PT})$, and it is expected that the same mechanism reappears in other sectors of Nature. For example, this mechanism is called for as a solution to the strong CP-problem of QCD, leading to the hypothesis that a new light state, called axion, would couple to SM particles.

And for many new physics scenarios we design, especially if they involve new strong interactions, these (pseudo-)Goldstone bosons are present and couple to SM particles via interactions which show growth with energy, similar to the EFT case.

Indeed, if we denote the new light state as $a$ and assumed for simplicity to have pseudoscalar quantum numbers, the interaction with the SM could look like

$$
\frac{\alpha}{f_{a}} a F^{\mu v} \tilde{F}_{\mu v}
$$

where $F_{\mu \nu}$ denotes the field strength of any of the SM interactions and $\alpha$ is the strength. This interaction is dimension-five, grows with $\widehat{s}$, and is suppressed by the scale $f_{a}$, the breaking scale of the UV global symmetry which led to the existence of $a$. Apart from couplings to gauge bosons, these pseudo-Goldstone bosons could couple to fermions, with a strength typically suppressed by the fermion mass.

Experimentally, distinguishing a genuinely EFT excess from an axion-like excess would require a detailed understanding of the tail's behaviour, which is currently not possible due to the lack of statistics in the tails as well as enough precision in the theoretical determination of these distributions at high energy. Nevertheless, one could envision a future situation at high-luminosity LHC where an excess over the Standard Model would be firmly established. In this case, the detailed study of the distribution could allow us to disentangle both hypotheses.

2.3. Broad Resonances: Models of Towers of States. Apart from heavy and light resonances, one could also interpret tails in energy distributions in terms of very close-by resonances, so close that they form an almost continuum. There are many models which could produce such behaviour, from towers of resonances with the same quantum numbers to quasiconformal theories. In this section, we will propose some benchmarks for interpretation and in Section 3 provide a dictionary to relate to them.

2.3.1. Warped Extradimensions. The EFT framework is very popular for many reasons, including its generality and ability to be improved order-by-order in an expansion of momenta over the heavy masses. Yet, EFTs are based on the assumption that the new sector is at a scale well separated from the electroweak scale, hence losing ground in terms of understanding the origin of the electroweak sector, including the hierarchy problem.

This problem has taken up a lot of the community's minds for a long time and accordingly been rephrased in many ways. One particularly compelling view to solve the hierarchy problem comes from linking gravitational interactions with the SM, in particular assuming that the scale of strong gravitational effects is much lower than the perceived Planck scale, all the way down to the TeV scale, close to the electroweak scale. This can be achieved by postulating the existence of new, small dimensions of space.

These new theories of extradimensions and strong gravity at the $\mathrm{TeV}$ scale could take many forms, encoded in the choice for space-time geometry and localisation of the fields inside the extradimension. A very well-studied and successful framework is the so-called Randall-Sundrum (RS) model [11] or simply warped extradimensions. It is based on a setup with a single new dimension of space with a large curvature and a nonfactorisable metric

$$
d s^{2}=e^{-2 k R|y|} \eta_{\mu \nu} d x^{\mu} d x^{\nu}-R^{2} d y^{2}
$$




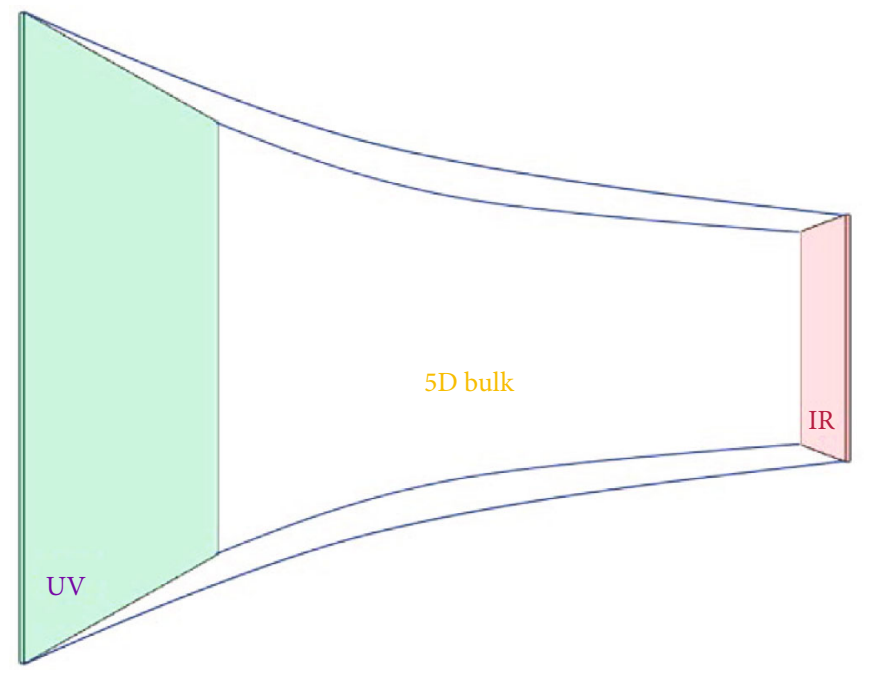

FIGURE 4: Schematic view of the RS geometry. The warp factor decreases exponentially along the bulk.

where $k$ is the $5 \mathrm{D}$ curvature, $2 \pi R$ is the size of the extradimension, and $y$ is the coordinate in the 5th dimension. The RS models is built on a slice of the 5D space-time located between two 4D branes, which are typically located at $y=0$ (UV-brane) and $y=\pi$ (IR-brane) (see Figure 4). The combination $e^{-2 k R|y|}$ is the warp factor and affects $4 \mathrm{D}$ slices of spacetime in different ways, exponentially different as we move along the bulk. In the classical $4 \mathrm{D}$ space-time, the gravitational fundamental interaction is the Planck mass, $\bar{M}_{p}=M_{p}$ $1 \sqrt{8 \pi}=2.435 \times 10^{18} \mathrm{GeV}$. The effect of the new extradimension is to modify the fundamental scale

$$
\bar{M}_{p}^{2}=\frac{M_{5}^{3}}{k}\left(1-e^{-2 k \pi R}\right)
$$

and to lower the scale of strong gravitational phenomena (like black hole formation) to the $\mathrm{TeV}$ scale near the IRbrane.

Warped models come in different varieties depending on particle localisations as well as the exact form of the geometry. Nevertheless, we generically expect the existence of towers of massive spin-2 resonances (Kaluza-Klein gravitons) as a consequence of compactifying higherdimensional gravity. Indeed, one can expand fluctuations around the $4 \mathrm{D}$ projection of the $5 \mathrm{D}$ metric to obtain

$$
G_{\mu \nu}^{(5)}=e^{-2 \sigma}\left(\eta_{\mu \nu}+\frac{2}{M_{5}^{3 / 2}} h_{\mu \nu}\right)
$$

where $h_{\mu \nu}$ can then be interpreted as a 5D graviton. After compactification, this $5 \mathrm{D}$ field can be written as a massless graviton and an infinite KK-tower of massive gravitons. The effective scale interaction of the KK-gravitons depends on the location in bulk of the field it interacts with. Fields near the IR couple to the KK-tower with scale $\Lambda \sim \mathrm{TeV}$, namely,

$$
\mathscr{L}=-\frac{1}{\Lambda_{\mathrm{RS}}} \sum_{n=1}^{\infty} h_{\mu \nu}^{n}(x) T^{\mu v}(x)
$$

where $T^{\mu v}$ is the SM energy-momentum tensor while $h_{\mu \nu}^{n}$ is the $n$th KK-graviton.

The spectrum of the KK-tower, how broad and spaced the states are, depends on the exact geometry, whether it takes the simple form of RS (Equation (5)) or something more complex. For example, if during compactification there were background fields in the bulk, the effective geometry could approximate RS near the UV-brane but deviate substantially near the IR $[12,13]$ and hence drastically changing the phenomenology.

In the simple case of RS, one can find the KK spectrum by solving the Einstein equation and the equation of motion for the KK-graviton:

$$
m_{n}=k x_{n} e^{-k \pi R}
$$

where $x_{n}$ are the roots of the $J_{1}$ Bessel function.

Finally, in RS or any other model of extradimensions, keeping a finite size for an extradimension (in our case, at $y$ $=\pi R$ ) is not trivial and usually relies on introducing new degrees of freedom. A popular mechanism of stabilisation is the Goldberger-Wise [14], which calls on a new 5D scalar that mixes with the graviscalar $\left(G_{55}\right)$, whose vev controls $R$, dynamically stabilising the size of the extradimension. This field can be written as a KK-tower, and its light zero mode is usually called radion (already mentioned in the EFT section). Its mass is a free parameter and interacts with SM fields via the trace of the stress tensor, $-1 / \sqrt{6} \Lambda_{\mathrm{RS}} r T_{\mu}^{\mu}$. As the strength of interactions is usually weaker than that of KKgravitons, in the rest of the paper, we only consider KK-gravitons, but the discussion could be extended to the radion. 


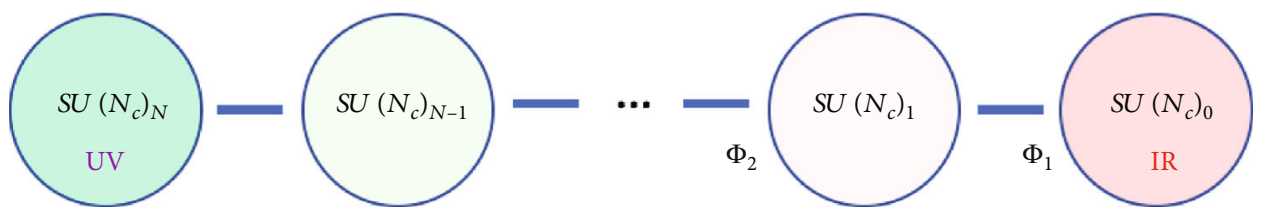

Figure 5: $S U\left(N_{c}\right)$ lattice representation of deconstruction.

2.3.2. Deconstruction. Towers of resonances can appear in many ways, not just as a consequence of the compactification of extradimensions. In this section, we discuss the first alternative interpretation of nonresonant phenomena produced by broad/nearby resonances. Our set-up is deconstruction [15], namely, the idea that certain types of gauge theories could resemble extradimensions as long as they provide a latticised version of a real (continuous) space coordinate (see Figure 5). The origin of deconstructed models goes far back to old models of mooses [16], series of new confining gauge sectors which at low energies exhibit interesting properties.

The simplest deconstruction framework assumes $N+1$ copies of $S U\left(N_{c}\right)$, where $N_{c}$ refers to a new QCD-type colour, and $N$ Higgs-like fields with a new $\operatorname{VEV}(v)$. The Lagrangian of these new fields can be written as

$$
\mathscr{L}=-\frac{1}{4} \sum_{i=1}^{N} F_{i \mu \nu}^{a} F^{i \mu v a}+\sum_{i=1}^{N} D_{\mu} \Phi_{i}^{\dagger} D^{\mu} \Phi_{i},
$$

where

$$
D_{i \mu}=\partial_{\mu}+i g N A_{i \mu}^{a} T_{i}^{a}
$$

and one typically assumes that all the $\Phi_{i}$ fields couple with the same coupling $(g)$.

These VEVs break $N+1 S U\left(N_{c}\right)$ symmetry to the diagonal $S U\left(N_{c}\right)$ gauge group. The mass-matrix for the gauge fields is given by

$$
\mathscr{M}=\frac{1}{2} g v\left(\begin{array}{cccccc}
1 & -1 & 0 & \ldots & 0 & 0 \\
-1 & 2 & -1 & \cdots & 0 & 0 \\
0 & -1 & 2 & \ldots & 0 & 0 \\
\vdots & \vdots & \vdots & \cdots & \vdots & \vdots \\
1 & 0 & 0 & \ldots & 2 & -1 \\
1 & -1 & 0 & \ldots & -1 & 1
\end{array}\right) \text {, }
$$

and the gauge field mass eigenstates can be obtained by diagonalizing this matrix. The mass for the $n$th gauge field can be written as

$$
M_{n}=2 g v \sin \left(\frac{n \pi}{2(N+1)}\right)
$$

which for $n \ll N$ approximates to

$$
M_{n} \approx \frac{g v \pi n}{N+1} .
$$

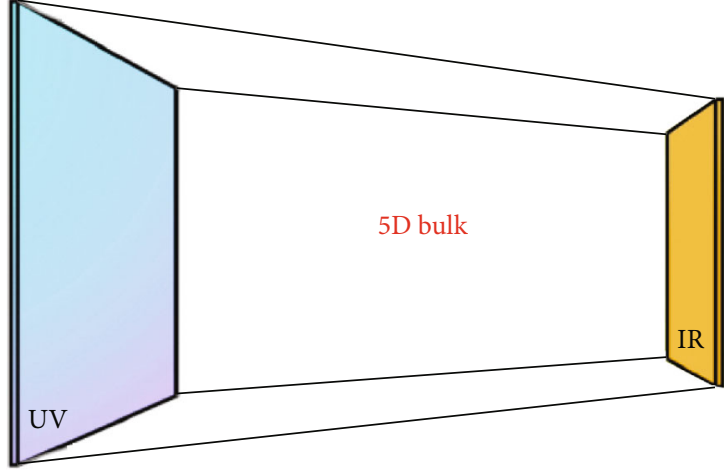

Figure 6: Schematic view of the Clockwork/Linear Dilaton model.

2.3.3. Clockwork/Linear Dilaton. Here, we discuss another example of models with limiting behaviour of broad/nearby resonances, clockwork models. They were born as a toy setup able to encapsulate some of the known phenomenology of extradimensions and deconstruction but allowing more flexibility of parameter choices. In particular, we will discuss one particular version of clockwork, the so-called continuum Clockwork/Linear Dilaton (CW/LD) model, which was proposed in Ref. [17] and whose nonresonant phenomenology has been studied in Ref. [18].

The metric that describes this scenario can be written as

$$
d s^{2}=e^{4 / 3 k R|y|}\left(\eta_{\mu \nu} d x^{\mu} d x^{\nu}-R^{2} d y^{2}\right)
$$

where the parameter $k$, called clockwork spring, represents the curvature along the 5th dimension and $R$, as in RS case, represents its size (see Figure 6).

There are many similarities with the RS framework, the compact 5D space-time also sandwiched between the UVbrane (at $y=0$ ) and IR-brane (at $y=\pi$ ). The SM could be localised in the 5D bulk, but in the minimal scenario, it is confined on the IR-brane. The relation between the fundamental $4 \mathrm{D}$ and $5 \mathrm{D}$ scales is given by

$$
\bar{M}_{p}^{2}=\frac{M_{5}^{3}}{k}\left(e^{2 \pi k R}-1\right) .
$$

In RS $M_{5} \sim \bar{M}_{p}$ and the interaction scale in the IR-brane is order TeV while in CW/LD $M_{5}$ is directly order TeV.

The mass spectrum of the $4 \mathrm{D}$ KK-gravitons tower is more spaced than in the RS case, as the masses for $n>0$ are given by 


$$
m_{n}^{2}=k^{2}+\frac{n^{2}}{R^{2}} .
$$

The effective interaction between the particles located at IR-brane and the KK-gravitons can be written as

$$
\mathscr{L}=-\sum_{n=1}^{\infty} \frac{1}{\Lambda_{n}} h_{\mu \nu}^{n}(x) T^{\mu \nu}(x) .
$$

Note that in these models, the effective interaction scale, $\Lambda_{n}$, depends on the mass of the KK-graviton; hence, each graviton couples differently to the brane particles

$$
\frac{1}{\Lambda_{n}}=\frac{1}{\sqrt{M_{5}^{3} \pi R}}\left(1-\frac{k^{2}}{m_{n}^{2}}\right)^{1 / 2},
$$

except the zero mode that, as in the RS framework, couples with $\Lambda_{0}=\bar{M}_{p}$.

In order to stabilise the size of the extradimension, $R$, in the CW/LD is common to place a dilaton field in the bulk. The spectrum of the dilaton's KK-tower is given by

$$
\begin{gathered}
m_{r}=m_{\Phi_{0}}=\frac{9}{8} k^{2}, \\
m_{\Phi_{n}}=k^{2}+\frac{n^{2}}{R^{2}},
\end{gathered}
$$

and the zero mode is identified as the radion field.

2.3.4. Other Models: Unparticles and Other Confining Sectors. We have described three scenarios with towers of resonances, which in some limits in the parameter space would lead to an almost continuum of broad/nearby resonances. In Section 3, we will discuss what this parameter space is.

Before moving onto the phenomenological aspects, we will discuss other theories which could mimic the phenomenology of warped extradimensions.

In the context of string theory, there is a huge body of work describing the idea that theories with weak gravity can be dual to strong gauge theories in a lower-dimensional space, inspired by the so-called AdS/CFT [19]. Indeed, those dualities between two types of theories seem to extend beyond the specific example of the AdS/CFT set-up. To some qualitative level, one can understand models like RS as duals to a $4 \mathrm{D}$ strongly coupled theory, where the KK-tower is interpreted as bound states of preon quark and gluons with increasing mass but the same quantum numbers. In QCD, we do observe to a certain extent a tower of resonances for each $J^{C P}$ choice; for example, in the vector sector, we have observed the $\rho$ meson, higher up in mass the $\rho^{\prime}$, followed by a continuum of broader $\rho^{n}$. The $J^{C P}$ properties of $\rho, \rho^{\prime}$ and the continuum can be derived from the angular distributions of the final states.

In this context, the KK-graviton in RS is no exception: it can be interpreted as Pauli-Fierz spin-two bound state of new quark and gluon preons. Indeed, it has been shown that the collider properties of the KK-graviton are indistinguishable from a hypothesised spin-two field from a new strongly coupled sector [20].

There are other possible alternatives to interpret this continuum, all closely related by dualities. For example, in Ref. [21], it has been proposed that black hole dynamics can lead to spin-two hair and that for low-energy strong gravity models, these spin-two states could be produced at colliders [22].

Yet another alternative interpretation of a continuous spectrum is provided by the so-called unparticles [23]. These theories are based on the observation that a quasiconformal sector coupled to the SM would show up at low energies as a continuous emission of energy, producing then a nonresonant tail on some distributions of SM particles.

To summarise, any interpretation of nonresonant phenomena in terms of RS parameters, or deconstructed, or clockwork, could be recasted as certain models of strongly coupled gauge theories, quantum black hole production, or a new quasiconformal sector in Nature.

\section{Phenomenological Signatures of Nonresonant Models}

We now move onto the phenomenological description of the models described in the previous section.

For the EFT case, the interpretation procedure is quite straightforward and already in the experiment's roadmap. There is a classification of possible deviations from the SM which could lead to excesses in tails, at $O\left(p^{2} / M^{2}\right)$ [24] and to some extent at $O\left(p^{4} / M^{4}\right)$ [25-27].

For the pseudo-Goldstone case, only recently, the nonresonant opportunities have been explored in Ref. [2], where it was noted that for light particles whose resonant region is not accessible due to selection cuts, tails may be the only way to discover those states.

Regarding broad/nearby resonances, in Ref. [18], a description of nonresonant diphoton analysis was done in the context of Clockwork/Linear Dilaton.

In this section, we are going to explore under what circumstances our scenarios would lead to nonresonant phenomena. Our aim is to provide a simplified framework to interpret these tails in terms of the three scenarios: heavy, light, and broad. For concreteness, we will focus on a simple final state, the dijet, yet the conclusions can be generalized to other final states. We will start with a parton-level discussion to move onto a more realistic hadron collider simulation.

\subsection{Parton-Level Cross-Sections}

3.1.1. The Heavy and Light Cases. In the partonic crosssection $(\widehat{\sigma})$, EFT effects would primarily appear as powers of $\widehat{s} / M^{2}$, where $M$ is the scale of new heavy resonances, namely, through the interference of EFT with SM amplitudes:

$$
\frac{\widehat{\sigma}-\widehat{\sigma}_{S M}}{\widehat{\sigma}_{S M}} \propto \bar{c} \frac{\widehat{s}}{M^{2}}
$$

which leads to a growing amplitude with the energy of the event. For the dijet case, there are a number of dimension- 6 
operators which could modify this cross-section, notably the pure gauge $c_{3 G} G_{\mu}^{\alpha} G_{\alpha}^{\beta} G_{\beta}^{\mu}$.

On the other hand, if the EFT operator is CP-odd, e.g., $\tilde{c}_{3 G} G_{\mu}^{\alpha} G_{\alpha}^{\beta} \tilde{G}_{\beta}^{\mu}$, the partonic cross-section would be further suppressed as the leading contribution would come from the $\mathrm{EFT}^{2}$ terms [28]:

$$
\frac{\widehat{\sigma}-\widehat{\sigma}_{S M}}{\widehat{\sigma}_{S M}} \propto \tilde{c}^{2} \frac{s \wedge^{2}}{M^{4}}
$$

which is steeper with $\widehat{s}$ and conversely more suppressed with the scale of the heavy resonances $M$. Note that beyond $\mathrm{CP}$ properties, the interference with the SM could vanish in a number of situations (see, e.g., Ref. [29]).

Interestingly, for a light pseudo-Goldstone boson, an axion-like particle, the off-shell production is quite similar to square EFT effects [2]. In the off-shell region, the partonic cross-section looks like

$$
\frac{\widehat{\sigma}-\widehat{\sigma}_{S M}}{\widehat{\sigma}_{S M}} \propto \frac{s \wedge^{2}}{M^{4}},
$$

so the growth with energy is also $E^{4}$ relative to the SM.

Hence, one can relate the EFT quadratic effects and axion-like parameters as follows:

$$
f_{a} \simeq \frac{M}{\sqrt{\tilde{c}}}(\mathrm{ALP} \leftrightarrow \mathrm{EFT}) ;
$$

namely, the decay constant of a light pseudo-Goldstone boson $\left(m_{a} \ll f_{a}\right)$ plays the role of the scale of new heavy states and their coupling to SM particles.

3.1.2. The Broad Case: Matching of Warped, Clockwork, and Deconstructed Models. The LHC has placed strong limits on resonances coupled to SM particles, often in the multi-TeV range. Now, imagine that these resonances were not isolated but came in a tower of multiple, close-by resonances, forming an almost continuum. In that case, usual bump searches would fail but also broad resonance searches, where broad often means $\Gamma / m>0.1$ and do not capture a quasicontinuum. In this case, only searches for excesses in tails could unveil their presence.

In the previous section, we have discussed many models which predict a spectrum with towers of states with the same quantum numbers. Here, we are going to discuss which regions of their parameter space brings these spaced towers close into a continuum. We will also obtain a dictionary among the three standard interpretations we discussed: warped extradimensions, clockwork, and deconstruction.

First of all, it is important to identify the free independent parameters of these three models. In the RS and CW/LD, the fundamental free parameters are the curvature along the fivedimension $k$ and the size $R$. In deconstruction, they are the number of sites $(N)$ and the scale times coupling of the inter-
TABLE 1: Free parameters of warped extradimensions (RS), Clockwork/Linear Dilaton, and deconstruction. The first parameter set represents the fundamental parameters of each model. The second parameter set shows the parameters that are useful for phenomenology (mass gap and coupling).

\begin{tabular}{lccc}
\hline & $\mathrm{RS}$ & $\mathrm{CW} / \mathrm{LD}$ & Decon. \\
\hline Fundamental parameters & $k_{\mathrm{RS}} R$ & $k_{\mathrm{CW}} R$ & $N g v$ \\
Useful parameters & $m_{1} \Lambda_{\mathrm{RS}}$ & $k_{\mathrm{CW}} M_{5 \mathrm{CW}}$ & $M_{1} g v$ \\
\hline
\end{tabular}

actions $(g v)$. Nevertheless, the fundamental parameters are not the best option in order to study the phenomenology, as we show in Table 1, but the mass gap and coupling to SM particles.

Despite their differences, one should be able to match these three models in the nonresonant limit, as the continuum is roughly characterized by two parameters, the threshold (mass gap) and the height of the spectrum.

We can start our matching exercise with the RS and CW/LD models. In the RS framework, the mass gap between two consecutive resonances can be found using Equation (9):

$$
\Delta m^{\mathrm{RS}} \approx \frac{m_{1} \pi}{x_{1}}
$$

where $m_{1}$ is the first graviton mass and we are assuming $n \gg 1$. In the CW/LD case, the masses are given by Equation (17), leading to

$$
\Delta m^{\mathrm{CW}} \approx \frac{1}{R}
$$

in the $k \ll(n / R)^{2}$ limit. In the CW/LD model, each graviton couple is different to the energy-momentum tensor (Equation (20)). In the $m_{n} \gg m_{1}$ limit (where the spectrum would approximate a continuum), the expression reduces to

$$
\Lambda_{\mathrm{CW}} \approx M_{5}^{3 / 2} \sqrt{\pi R} .
$$

The matching between the two spectra can be obtained by equating Equations (26) and (27), which leads to the following relations:

$$
\begin{gathered}
R \approx \frac{x_{1}}{\pi m_{1}}(\mathrm{CW} / \mathrm{LD} \leftrightarrow \mathrm{RS}), \\
M_{5_{\mathrm{CW}}}=\left(\frac{\Lambda_{\mathrm{RS}}}{\sqrt{\pi R}}\right)(\mathrm{CW} / \mathrm{LD} \leftrightarrow \mathrm{RS}) .
\end{gathered}
$$

Now, using Equation (17), one can obtain the equivalent $k_{\mathrm{CW}}$ value.

In Figure 7, we can see an example of bringing both theories to the broad/nearby resonance limit and performing this matching. The interactions of KK-resonances to SM 


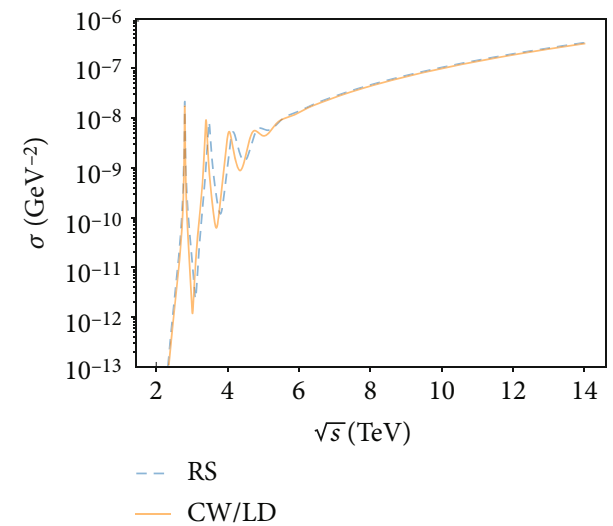

Figure 7: Comparison between the RS (dashed-blue line) and the CW (solid-orange line) models in the matching region. For this plot, we chose $m_{1}=2000 \mathrm{GeV}$ and $\Lambda_{\mathrm{RS}}=5100 \mathrm{GeV}$ which implies $M_{5_{\mathrm{CW}}} \approx 2390 \mathrm{GeV}$ and $k_{\mathrm{CW}} \approx 1131 \mathrm{GeV}$.

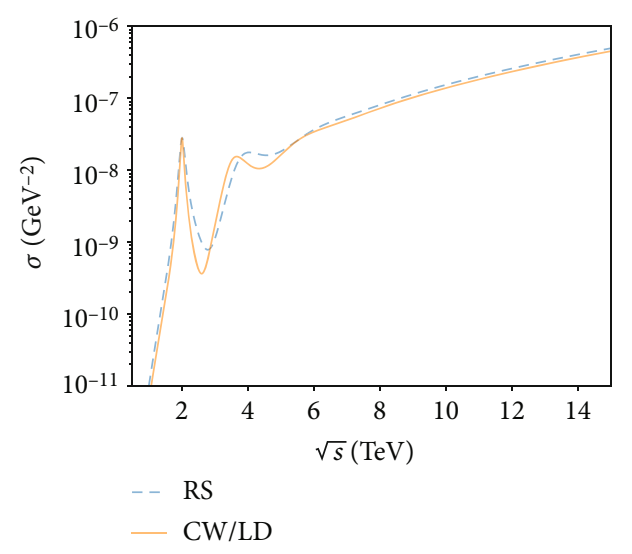

FIGURE 8: Comparison between the RS (dashed-blue line) and the CW (solid-orange line). For this plot, we choose $\lambda=1.5$ and NP $=$ 1, i.e., $m_{1}=2000 \mathrm{GeV}, \Lambda_{\mathrm{RS}}=2128 \mathrm{GeV}, M_{5_{\mathrm{CW}}} \approx 1334 \mathrm{GeV}$, and $k_{\mathrm{CW}} \approx 1131 \mathrm{GeV}$.

particles are of the form given in Equation (8), hence grow with energy as

$$
\widehat{\sigma} \propto \frac{\widehat{s}}{\Lambda_{\mathrm{RS}}^{2}} .
$$

We can also bring the continuum spectrum closer to the first resonance. This situation occurs when $\Delta m=\lambda^{2} \times \Gamma_{1}$ (where $\lambda$ is $\mathcal{O}(1)$ parameter). In this particular region, the width of the resonances is more or less the value of the gap, and we obtain an overlapping between the resonances. The total decay of the first graviton is

$$
\Gamma_{n}=\frac{m_{n}^{3}}{\pi \Lambda_{\mathrm{RS}}^{2}}\left(\frac{73}{240}+\mathrm{NP}\right),
$$

where NP represents the new physics contributions (possible decays to non-SM final states). Assuming this information, we can write the condition for broadness as

$$
\Lambda_{\mathrm{RS}}=\lambda \frac{m_{1}}{\pi} \sqrt{\left(\frac{73}{240}+1\right) x_{1}} .
$$

In Figure 8, we show an example for this kind of spectrum, where we considered $\mathrm{NP}=1$ (the same order than SM contribution) while $\lambda=1.5$, chosen so that there is no conflict with the $\Lambda_{\mathrm{RS}}>m_{1}$ condition.

We have shown the relation between RS and CW/LD in the nonresonant regime. The same exercise can be done for deconstruction models. One can easily find that the deconstruction fundamental parameters are related to RS parameters as follows:

$$
\begin{gathered}
g v=\Lambda_{\mathrm{RS}}(\text { Decon. } \leftrightarrow \mathrm{RS}), \\
N+1=\frac{x_{1} \Lambda_{\mathrm{RS}}^{2}}{\bar{M}_{p l} m_{1}} \ln \left(\frac{\bar{M}_{p l}}{\Lambda_{\mathrm{RS}}}\right) .
\end{gathered}
$$

3.2. Nonresonant Tails at the Large Hadron Collider. In the previous section, we have discussed the differences among the three scenarios at the parton level:

(i) EFT, with interference with SM: grows as $\widehat{s} / M^{2}$

(ii) Off-shell light axion-like: grows as $s \wedge^{2} / f_{a}^{4}$

(iii) Broad/nearby resonances: quick increase from the mass gap location, grows as $\widehat{s} / \Lambda^{2}$ after that

Now, we compute numerically the total cross-section (including hadronization and PDF effects) at LHC energies. The results are shown in Figure 9, where one can observe the hierarchies we just described: the strongest slope comes from the off-shell axion-like $s \wedge^{2}$ behaviour, followed by the EFT $\widehat{s}$. The broad resonance case overcomes the mass gap to follow with the $\widehat{s}$ slope of dimension-five interactions. We also plot the SM dijet production for comparison.

We model the tower of broad resonances with a simple modification of an existing Monte Carlo model provided in Ref. [30] and based on the implementation of spin-two resonances in Ref. [31]. The resonances start at $2 \mathrm{TeV}$ and are set to be nearby, $\Delta m_{i j} \sim \Gamma_{i, j}$. We have set the coupling of all resonances to be the same, as in the RS models. As we are plotting signal-only normalized to the total cross-section, the specific value of $\Lambda_{\mathrm{RS}}$ is not relevant.

The EFT example has been computed using the Monte Carlo implementation in Ref. [3] and with operators enabling new four- and three-gluon vertices such as

$$
\frac{g_{s}^{3} \bar{c}_{3 G}}{m_{W}^{2}} f_{a b c} G_{\mu \nu}^{a} G_{\rho}^{b, v} G^{c, \rho \mu}
$$

where we set $\bar{c}_{3 G}=0.1$ and plotted the SM+EFT effect, including the dominant interference. 


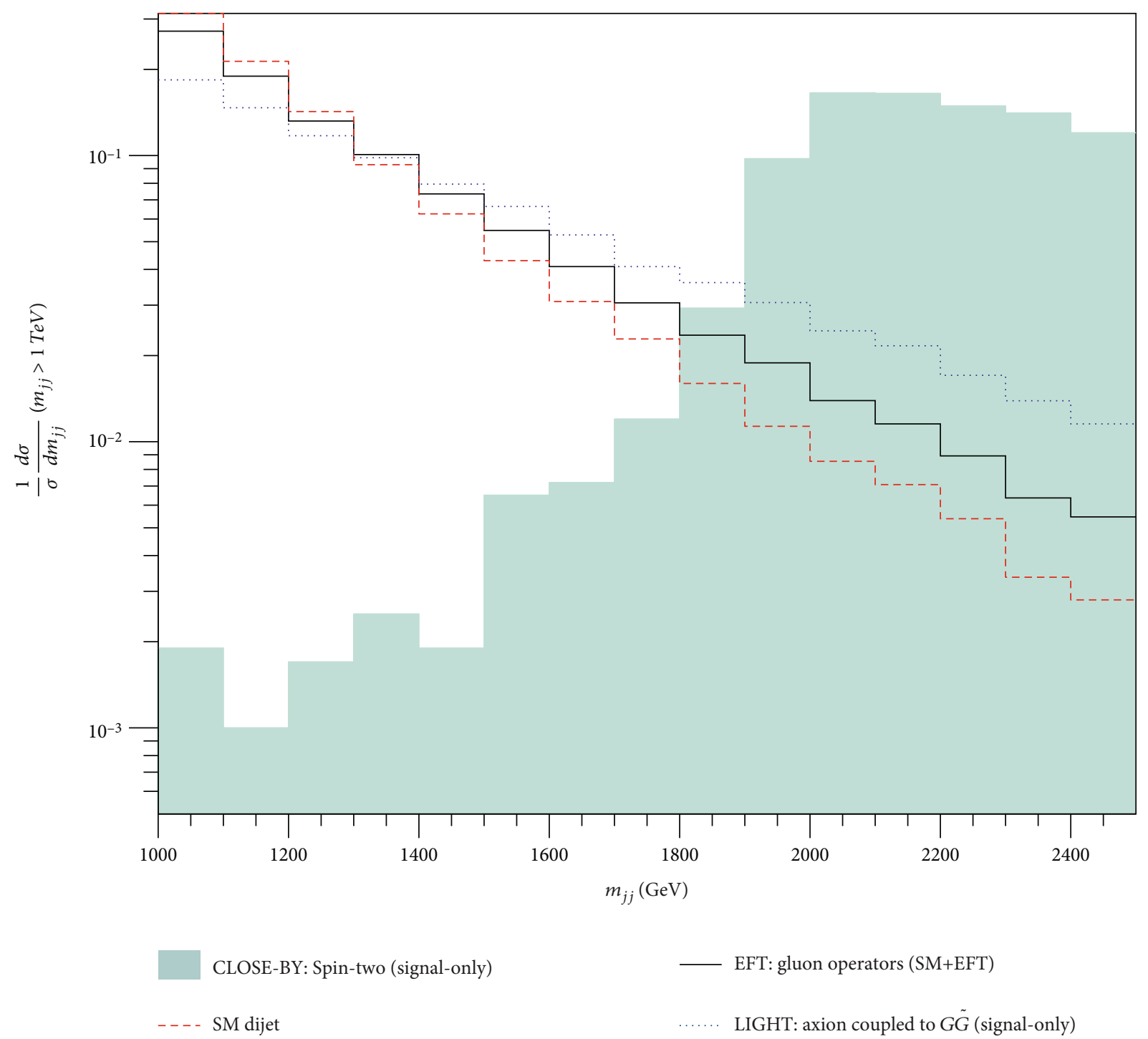

FIGURE 9: The $m_{j j}$ differential distribution in the SM, EFT, axion-like, and broad resonance cases.

For the axion-like case, we used the Monte Carlo implementation from the study in Ref. [32] to compute the offshell axion exchange. As for the RS model, the specific value of $f_{a}$ is not relevant for this normalized distribution.

\section{Outlook}

In the current state of affairs at the LHC, the EFT framework is gaining support as a benchmark for interpretation of nonresonant phenomena. Within this framework, traditional searches for new physics, resonant searches, are replaced by searches for extended excesses, typically in the high- $p_{T}$ tails of SM distributions.

The EFT is by no means the only way to think of nonresonant behaviour. Many scenarios, at least in some area of their parameter space, can only be explored in the nonresonant kinematic regions.
In this paper, we have described many of these types of models, classify them in terms of heavy, light, or broad/nearby particles, and have given a dictionary among them. For example, we have related parameters of axion-like models with EFT effects, and warped extradimensions with clockwork and deconstruction models.

Our aim was to provide a framework for reinterpretation of nonresonant limits and for future characterization of a possible confirmed excess. Our theory examples represent a diverse set of ideas in our area and include axion-like particles, extradimensional KK-resonances, unparticles, deconstruction, clockwork, quantum black holes, and strong-coupling gauge duals of gravity theories.

We have discussed the parton- and hadron-level behaviour of dijet distributions, but all these models could be explored with the same detailed study of kinematic distributions of various final states, including missing energy $+X$, massive diboson, Higgs $+Z$, and di-Higgs. 
We hope that this work will motivate a broader view of the physics potential of collider nonresonant searches.

\section{EndNotes}

See Refs. [33-35] for examples on how background fields can change the spectrum of KK states. See Ref. [36] for a recent discussion on the phenomenology of this operator and Ref. [37] for a canonical reference.

\section{Data Availability}

The paper is based on simulated data which can be obtained by contacting the authors.

\section{Conflicts of Interest}

The authors declare that they have no conflicts of interest.

\section{Acknowledgments}

V.S. acknowledges support from the UK Science and Technology Facilities Council ST/L000504/1.

\section{References}

[1] ATLAS collaboration, "Measurement of fiducial and differential $W^{+} W^{-}$production cross-sections at $\sqrt{s}=13 \mathrm{TeV}$ with the ATLAS detector," The European Physical Journal C, vol. 79, no. 10, p. 884, 2019.

[2] M. B. Gavela, J. M. No, V. Sanz, and J. F. de Troconiz, "Nonresonant searches for axionlike particles at the LHC," Physical Review Letters, vol. 124, no. 5, article 051802, 2020.

[3] A. Alloul, B. Fuks, and V. Sanz, "Phenomenology of the Higgs effective Lagrangian via FEYNRULES," Journal of High Energy Physics, vol. 2014, no. 4, article 110, 2014.

[4] M. Gorbahn, J. M. No, and V. Sanz, "Benchmarks for Higgs effective theory: extended Higgs sectors," Journal of High Energy Physics, vol. 2015, no. 10, article 36, 2015.

[5] E. Masso and V. Sanz, "Limits on anomalous couplings of the Higgs boson to electroweak gauge bosons from LEP and the LHC," Physical Review D, vol. 87, no. 3, article 033001, 2013.

[6] K. Mimasu, V. Sanz, and C. Williams, "Higher order QCD predictions for associated Higgs production with anomalous couplings to gauge bosons," Journal of High Energy Physics, vol. 2016, no. 8, article 39, 2016.

[7] J. Brehmer, A. Freitas, D. Lopez-Val, and T. Plehn, "Pushing Higgs effective theory to its limits," Physical Review D, vol. 93, no. 7, article $075014,2016$.

[8] R. Contino, A. Falkowski, F. Goertz, C. Grojean, and F. Riva, "On the validity of the effective field theory approach to SM precision tests," Journal of High Energy Physics, vol. 2016, no. 7, article 144, 2016.

[9] A. Biekotter, J. Brehmer, and T. Plehn, "Extending the limits of Higgs effective theory," Physical Review D, vol. 94, no. 5, article 055032, 2016.

[10] C. Degrande, B. Fuks, K. Mawatari, K. Mimasu, and V. Sanz, "Electroweak Higgs boson production in the standard model effective field theory beyond leading order in QCD," The European Physical Journal C, vol. 77, no. 4, p. 262, 2017.
[11] L. Randall and R. Sundrum, "Large mass hierarchy from a small extra dimension," Physical Review Letters, vol. 83, no. 17 , pp. 3370-3373, 1999.

[12] J. Hirn and V. Sanz, "Interpolating between low and high energy QCD via a 5-D Yang-Mills model," Journal of High Energy Physics, vol. 2005, no. 12, article 030, 2005.

[13] J. Hirn and V. Sanz, "NegativeSparameter from holographic technicolor," Physical Review Letters, vol. 97, no. 12, article 121803, 2006.

[14] W. D. Goldberger and M. B. Wise, "Modulus stabilization with bulk fields," Physical Review Letters, vol. 83, no. 24, article 4922, 4925 pages, 1999.

[15] N. Arkani-Hamed, A. G. Cohen, and H. Georgi, "(De)constructing dimensions," Physical Review Letters, vol. 86, no. 21 , article 4757, 4761 pages, 2001.

[16] H. Georgi, "A tool kit for builders of composite models," Nuclear Physics B, vol. 266, no. 2, pp. 274-284, 1986.

[17] G. F. Giudice and M. McCullough, "A clockwork theory," Journal of High Energy Physics, vol. 2017, no. 2, article 36, 2017.

[18] G. F. Giudice, Y. Kats, M. McCullough, R. Torre, and A. Urbano, "Clockwork/linear dilaton: structure and phenomenology," Journal of High Energy Physics, vol. 2018, no. 6, article 9, 2018.

[19] J. Maldacena, "The large N limit of superconforma field theories and supergravity," International Journal of Theoretical Physics, vol. 38, no. 4, pp. 1113-1133, 1999.

[20] R. Fok, C. Guimaraes, R. Lewis, and V. Sanz, "It is a graviton! Or maybe not," Journal of High Energy Physics, vol. 2012, no. 12, article 062, 2012.

[21] G. Dvali, "Black holes with quantum massive spin-2 hair," Physical Review D, vol. 74, no. 4, article 044013, 2006.

[22] G. Dvali and M. Redi, "Black hole bound on the number of species and quantum gravity at CERN LHC," Physical Review $D$, vol. 77, no. 4, article 045027, 2008.

[23] H. Georgi, "Unparticle physics," Physical Review Letters, vol. 98, no. 22, article 221601, 2007.

[24] B. Grzadkowski, M. Iskrzynski, M. Misiak, and J. Rosiek, "Dimension-six terms in the standard model Lagrangian," Journal of High Energy Physics, vol. 2010, no. 10, article 085, 2010.

[25] C. Hays, A. Martin, V. Sanz, and J. Setford, "On the impact of dimension-eight SMEFT operators on Higgs measurements," Journal of High Energy Physics, vol. 2019, no. 2, article 123, 2019.

[26] C. W. Murphy, "Dimension-8 operators in the standard model effective field theory," 2020, https://arxiv.org/abs/2005.00059.

[27] H.-L. Li, Z. Ren, J. Shu, M.-L. Xiao, J.-H. Yu, and Y.-H. Zheng, "Complete set of dimension-8 operators in the standard model effective field theory," 2020, https://arxiv.org/abs/2005.00008.

[28] F. Ferreira, B. Fuks, V. Sanz, and D. Sengupta, "Probing $C P$ -violating Higgs and gauge-boson couplings in the standard model effective field theory," The European Physical Journal C, vol. 77, no. 10, p. 675, 2017.

[29] A. Azatov, R. Contino, C. S. Machado, and F. Riva, "Helicity selection rules and noninterference for BSM amplitudes," Physical Review D, vol. 95, no. 6, article 065014, 2017.

[30] N. D. Christensen and C. Duhr, "FeynRules - Feynman rules made easy," Computer Physics Communications, vol. 180, no. 9, pp. 1614-1641, 2009. 
[31] K. Hagiwara, J. Kanzaki, Q. Li, and K. Mawatari, "HELAS and MadGraph/MadEvent with spin-2 particles," The European Physical Journal C, vol. 56, no. 3, pp. 435-447, 2008.

[32] I. Brivio, M. B. Gavela, L. Merlo et al., "ALPs effective field theory and collider signatures," The European Physical Journal C, vol. 77 , no. 8 , p. 572, 2017.

[33] J. Hirn, N. Rius, and V. Sanz, "Geometric approach to condensates in holographic QCD,” Physical Review D, vol. 73, no. 8, article 085005, 2006.

[34] J. Hirn, A. Martin, and V. Sanz, "Benchmarks for new strong interactions at the LHC," Journal of High Energy Physics, vol. 2008, no. 5, article 084, 2008.

[35] J. Hirn, A. Martin, and V. Sanz, "Describing viable technicolor scenarios," Physical Review D, vol. 78, no. 7, article 075026, 2008.

[36] R. Goldouzian and M. D. Hildreth, "LHC dijet angular distributions as a probe for the dimension-six triple gluon vertex," 2020, https://arxiv.org/abs/2001.02736.

[37] L. Dixon and Y. Shadmi, "Testing gluon self-interactions in three-jet events at hadron colliders," Nuclear Physics B, vol. 423, no. 1, pp. 3-32, 1994. 